\title{
Preoperative evaluation of the degree of liver fibrosis based on matter-element analysis using serological indicators in patients with hepatocellular carcinoma
}

\author{
Wei Liu ${ }^{1,}$, Xiaosong Li $^{1, \S}$, Weihong Zheng ${ }^{2}$, Rucheng Yao ${ }^{1}$, Jun Zheng ${ }^{1, *}$ \\ ${ }^{1}$ Department of Hepatopancreatobilary Surgery, The First College of Clinical Medical Sciences, Three Gorges University, \\ Yichang, Hubei, China: \\ ${ }^{2}$ Department of Pharmacology, Medical Science College, Three Gorges University, Yichang, Hubei, China.
}

\begin{abstract}
Summary Evaluation of the degree of liver fibrosis is an important basis for the clinical diagnosis and treatment of patients with hepatocellular carcinoma (HCC). It is meaningful to make a preoperative evaluation with non-invasive methods. In the current study, 12 commonly used preoperative serological indicators from $161 \mathrm{HCC}$ patients with different degree of liver fibrosis were collected retrospectively, and 8 of the indicators (ALB, PA, TBil, INR, AST, GGT, ALP, and PT) were ultimately used in matter-element analysis to create a formula. The relationship between those results and the histological sub-classification of the Laennec liver fibrosis scoring system was analyzed. The calculated value of $\mathbf{R}$ from this formula will indicate the differing degree of liver fibrosis in a patient: $i$ ) the value of $0.802 \leq \mathrm{R}<1$ indicates the early stage of liver cirrhosis, which corresponds to Laennec stages $0-3$; $i$ i) the value of $0.752 \leq R<0.802$ indicates the mild stage of liver cirrhosis, which corresponds to Laennec stage $4 \mathrm{~A}$; iii) the value of $0.698 \leq R<0.752$ indicates the moderate stage of liver cirrhosis, which corresponds to Laennec stage $4 \mathrm{~B}$; and $i v$ ) the value of $0.444 \leq \mathrm{R}<0.698$ indicates the severe stage of liver cirrhosis, which corresponds to Laennec stage $4 \mathrm{C}$. The hope is that this formula for preoperative evaluation of the degree of liver fibrosis using non-invasive methods would be useful in the clinical diagnosis and treatment of patients with $\mathrm{HCC}$ in the future.
\end{abstract}

Keywords: Liver cirrhosis, Laennec system, matter-element analysis

\section{Introduction}

Cirrhosis is defined as regenerative nodules surrounded by extensive fibrosis (1). Liver fibrosis is a pathological process of abnormal proliferation of connective tissues caused by various pathogenic factors, in which excessive extracellular matrix proteins accumulate in the liver. Among the various causes of liver fibrosis, viral hepatitis, alcoholic liver, fatty liver, and autoimmune diseases are the most common in clinical settings (2-4). Without proper treatment, liver fibrosis

\footnotetext{
${ }^{\S}$ These authors contributed equally to this work.

*Address correspondence to:

Dr. Jun Zheng, Department of Hepatopancreatobilary Surgery, The First College of Clinical Medical Sciences, Three Gorges University, No. 183 Yiling Road, Yichang, Hubei 443003., China.

E-mail: zhengjun1995@163.com
}

can eventually develop into cirrhosis and hepatocellular carcinoma (HCC) with the progression of the disease (5-7). Therefore, evaluation of the degree of liver fibrosis is an important basis for the clinical diagnosis and treatment of patients with the disease.

For semi-quantitative estimation of fibrosis, the Laennec system (Table 1) that is based on histological parameters of fibrous septa according to their width and number has been proposed. The Laennec system subdivides the most severe stage of fibrosis (F4) into $4 \mathrm{~A}, 4 \mathrm{~B}$, and $4 \mathrm{C}$ in order to acknowledge the varying severity of cirrhosis (8-10). However, the Laennec system is based on histological parameters of fibrous septa. Preoperative evaluation of the degree of liver fibrosis based on a simple and effective non-invasive method would prove beneficial.

The search for disease-related biomarkers in plasma has made rapid progress over the past few years, and the 
significance of biomarkers in diagnosis of disease has been widely demonstrated (11). Determining a useful method of evaluating the degree of liver fibrosis with serological biomarkers would be of great significance.

Various scoring formulae have been devised to evaluate HCC with serological biomarkers (12). A previous study by the current authors found that a scoring formula for liver injury (SFLI) was useful in the assessment of liver damage (13). SFLI was based on matter-element analysis. In the current study, matterelement analysis was used to create a formula based on serological indicators to facilitate preoperative evaluation of the degree of liver fibrosis in patients with HCC. The relationship between those results and the histological sub-classification of the Laennec system was analyzed.

\section{Materials and Methods}

\subsection{Study population}

From June 2010 to June 2015, 362 patients with HCC were treated in the Department of Liver Surgery of Yichang Central People's Hospital; 300 of those patients were diagnosed with different degrees of liver fibrosis. Patients under 30 years of age or over 70 years of age were excluded, patients with active hepatitis, patients using immunosuppressive or antiviral drugs, and patients with $\mathrm{HIV}, \mathrm{HCV}$, decompensation of the liver, alcoholic hepatitis, or an autoimmune disease were also excluded. Ultimately, 161 patients were enrolled in this study.

Data such as the patient's medical history, results of physical examinations, results of B-mode ultrasound, computed tomography (CT) scans, magnetic resonance imaging (MRI) scans, biochemistry, and results of postoperative pathology were retrospectively collected for all 161 patients. Paraffin-embedded specimens were obtained from Yichang Central People's Hospital for research purposes. This study was approved by the Ethics Committee of the First Clinical Medicine College Hospital of China Three Gorges University.

\subsection{Collection of data on serological indicators}

Data on the following 12 commonly used serological indicators were collected in this study: serum albumin (ALB), prealbumin (PA), serum total bilirubin (TBiL), the international normalized ratio (INR), aspartate aminotransferase (AST), gamma-glutamyl transpeptidase (GGT), alkaline phosphatase (ALP), prothrombin time (PT), serum creatinine ( $\mathrm{SCr}$ ), alanine aminotransferase (ALT), activated partial thromboplastin time (APTT), and thrombin time (TT). The serological indicators were collected in the first morning when the patients visited the doctors.

The normal values for these indicators were: ALB,
35-55 g/L; PA, 100-400 mg/L; TBiL, 2.04-20.4 $\mu \mathrm{mol} /$ L; INR, 0.8-1.5; AST, 0-40 U/L; GGT, 0-54 U/L; ALP, 39-117 U/L; PT, 11-13s; SCr, 70-106 $\mu \mathrm{mol} / \mathrm{L}$; ALT, 0-40 U/L; APTT, 28-41s; TT, 13-18s.

In order to comply with the statistical principles of matter-element analysis, the 161 patients were divided into 8 groups by age: $30-34,35-39,40-44,45-49,50-$ 54, 55-59, 60-64, and 65-69.

\subsection{Pathological grading according to the Laennec system}

After they were embedded in paraffin, 161 liver tissue specimens were cut into sections. Masson trichrome staining was performed as usual. All pathological sections were graded independently by two pathologists in this Hospital, the samples with the same result were selected and graded according to the Laennec liver fibrosis scoring system $(9,14)$.

In accordance with the histological sub-classification of the Laennec system (Table 1), liver fibrosis in 161 patients was divided into 7 degrees from mild to severe. Liver fibrosis was grades 0-3 (the early stage of liver cirrhosis) in 15 patients, grade $4 \mathrm{~A}$ (the mild stage of liver cirrhosis) in 42 , grade $4 \mathrm{~B}$ (the moderate stage of liver cirrhosis) in 55, and grade 4C (the severe stage of liver cirrhosis) in 49.

\subsection{Creation of a scoring formula using matter-element analysis}

In the process of creating a mathematical formula to score liver fibrosis, matter-element analysis was mainly used to solve for the weight of each detection index. The $\mathrm{R}$ value was calculated according to the fibrosis scoring formula. The method used was as follows:

(1) The age range of patients in the experiment was considered to be Tm, each measurement indicator was considered to be $\mathrm{Cn}$, and the obtained data were considered to be Xji $(j=1,2, \ldots, m ; i=1,2, \ldots, n)$. The following matter elements were successfully constructed:

$$
\mathrm{R}_{\mathrm{mm}}=\left|\begin{array}{ccccc} 
& \mathrm{T}_{1} & \mathrm{~T}_{2} & \ldots & \mathrm{T}_{\mathrm{m}} \\
\mathrm{C}_{1} & \mathrm{X}_{11} & \mathrm{X}_{21} & \ldots & \mathrm{X}_{\mathrm{m} 1} \\
\mathrm{C}_{2} & \mathrm{X}_{12} & \mathrm{X}_{22} & \ldots & \mathrm{X}_{\mathrm{m} 2} \\
\cdots & \ldots & \ldots & \ldots & \cdots \\
\mathrm{C}_{\mathrm{n}} & \mathrm{X}_{1 \mathrm{n}} & \mathrm{X}_{2 \mathrm{n}} & \ldots & \mathrm{X}_{\mathrm{mm}}
\end{array}\right|
$$

(2) Determination of the membership degree (U): in order to obtain the weight of each measurement indicator in the fibrosis scoring formula, a measurement standard should be determined. This standard was determined using membership degree (U), which was 
determined using the following method:

smaller measurement values indicated better results:

$$
\mathrm{U}_{\mathrm{ji}}=\frac{\max _{\mathrm{ji}}-\mathrm{X}_{\mathrm{ji}}}{\max _{\mathrm{ji}}-\min \mathrm{X}_{\mathrm{ji}}}
$$

greater measurement values indicated better results:

$$
\mathrm{U}_{\mathrm{ji}}=\frac{\mathrm{X}_{\mathrm{ji}}-\min \mathrm{X}_{\mathrm{ji}}}{\max \mathrm{X}_{\mathrm{ji}-\min \mathrm{X}_{\mathrm{ji}}}}
$$

where $\max \mathrm{Xji}$ and $\min \mathrm{Xji}$, represent the corresponding maximum and minimum values of $\mathrm{Xji}$ in each age group respectively.

(3) Conversion of the membership degree into relevance: the relevance conversion is the conversion between the degree of membership and the correlation coefficient. Since the correlation coefficient $(\xi)$ is equivalent to the membership function, the correlation coefficient $\xi j i$ can be determined from the membership coefficient Uji, namely: $\xi j i=U j i(j=1,2, \ldots, m ; i=1,2, \ldots, n)$.

(4) Establishment of the fuzzy matter element:

$\mathrm{R} \xi=\left|\begin{array}{ccccc} & \mathrm{P}_{1} & \mathrm{P}_{2} & \ldots & \mathrm{P}_{\mathrm{m}} \\ \mathrm{C}_{1} & \xi_{11} & \xi_{21} & \ldots & \xi_{\mathrm{m} 1} \\ \mathrm{C}_{2} & \xi_{12} & \xi_{22} & \ldots & \xi_{\mathrm{m} 2} \\ \ldots & \ldots & \ldots & \ldots & \ldots \\ \mathrm{C}_{n} & \xi_{1 \mathrm{n}} & \xi_{2 \mathrm{n}} & \ldots & \xi_{\mathrm{mn}}\end{array}\right|$

(5) Solving the weight for each indicator in the fibrosis scoring formula

$\mathrm{Wj}$ represents the weight for each indicator:

$$
\mathrm{W}_{\mathrm{j}}=\left|\begin{array}{ccc}
\mathrm{m} & \mathrm{m} & \mathrm{n} \\
\sum \xi_{\mathrm{ji}} / & \sum & \sum \xi_{\mathrm{ji}} \\
\mathrm{j}=1 & \mathrm{j}=1 & \mathrm{i}=\mathrm{l}
\end{array}\right|
$$

And

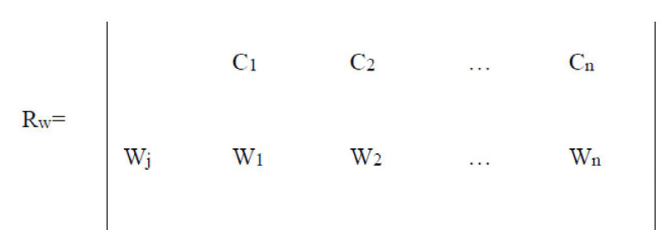

\subsection{Statistical analysis}

All of the determined indicators were used to construct a table, and Excel and the statistical software SPSS 18.0 were used to perform statistical analysis. The results are expressed as the mean $+\mathrm{SD}( \pm \mathrm{S})$. LSD and SNK were used when homogeneity of variance was present in group pair-wise comparisons, or Tamhane's T2 was more appropriate. ALB, PA, TBiL, SCr, INR, ALT, AST, gamma-GT, ALP, PT, and APTT were compared with their corresponding normal values. An Excel function was used to perform a $t$-test and the software SPSS18.0 was used to perform a $t$-test and analysis of variance. $p<0.05$ served as the level of significance, and $p<0.01$ indicated a significant difference.

The one-way analysis of variance was performed if the obtained data followed a normal distribution. Otherwise, the Kruskal Wallis test was used instead to analyze the correlation. In addition, Spearman rank correlation analysis was used in the correlation of all indexes and pathological diagnosis of the stage of liver fibrosis.

\section{Results}

\subsection{Screening on preoperative serological indicators}

Serological indicators and the stage of fibrosis were analyzed in the 161 patients according to the Laennec liver fibrosis scoring system (Table 1). Correlation analysis indicated that 11 of the 12 indicators were correlated with the Laennec liver fibrosis scoring system (Table 2), although some of the correlations were relatively weak ( $\mathrm{rs}<0.4)$. To optimize indicator selection, APTT and TT were excluded from analysis since these two indicators had a very low correlation. $\mathrm{SCr}$ was also excluded because its $\mathrm{p}$ value was greater than 0.05. Moreover, ALT was excluded since it belongs to the same system as AST, and AST is reported to be more strongly correlated with liver fibrosis than ALT (15).

Ultimately, 8 indicators - ALB, PA, TBiL, INR, AST, GGT, ALP, and PT - were selected for use in creating a formula using matter-element analysis.

\subsection{Creation of the liver fibrosis formula}

A database of the 8 preoperative serological indicators was created, and statistical analysis was performed using the statistical software SPSS18.0. Patients were divided into 8 age groups in increments of 5 years. Comparison between groups was performed with the range of reference values and the overall mean according to the t-test. A result greater than the upper limit or less than the lower limit was considered significant, with a test level $(\alpha)$ of 0.05 . Indicators resulting in significant differences were incorporated into the element of matter-element analysis, and the liver fibrosis formula was then created.

Liver fibrosis in the 161 patients was classified as grades $0,1,2,3,4 \mathrm{~A}, 4 \mathrm{~B}$, and $4 \mathrm{C}$. The 8 preoperative 
Table 1. The Laennec liver fibrosis scoring system in liver biopsies*

\begin{tabular}{|c|c|c|c|c|}
\hline Stage & Name & $\begin{array}{l}\text { Septa } \\
\text { (thickness and number) }\end{array}$ & Criteria & Score \\
\hline 0 & No definite fibrosis & $+/-$ & No definite fibrosis. & 0 \\
\hline 1 & Minimal fibrosis & + & $\begin{array}{l}\text { No septa or rare thin septum; may have portal expansion or mild } \\
\text { sinusoidal fibrosis. }\end{array}$ & 1 \\
\hline 2 & Mild fibrosis & ++ & $\begin{array}{l}\text { Occasional thin septa; may have portal expansion or mild } \\
\text { sinusoidal fibrosis. }\end{array}$ & 2 \\
\hline 3 & Moderate fibrosis & +++ & Moderate thin septa; up to incomplete cirrhosis. & 3 \\
\hline $4 \mathrm{~A}$ & Cirrhosis, mild, definite, or probable & ++++ & $\begin{array}{l}\text { Marked septation with rounded contours or visible nodules; Most } \\
\text { septa are thin (one broad septum allowed). }\end{array}$ & 4 \\
\hline 4B & Moderate cirrhosis & +++++ & $\begin{array}{l}\text { At least two broad septa, but no very broad septa and less than } \\
\text { half of biopsy length composed of minute nodules }\end{array}$ & 5 \\
\hline $4 \mathrm{C}$ & Severe cirrhosis & ++++++ & $\begin{array}{l}\text { At least one very broad septum or more than half of biopsy } \\
\text { length composed of minute nodules (micronodular cirrhosis) }\end{array}$ & 6 \\
\hline
\end{tabular}

*Histological sub-classification according to the Laennec system based on references $(9,14)$.

Table 2. Spearman correlation analysis between serum markers and the Laennec liver fibrosis scoring system

\begin{tabular}{lrl}
\hline Serum markers & \multicolumn{1}{c}{$\mathrm{r}_{\mathrm{s}}$} & $p$ value \\
\hline ALB $(\mathrm{g} / \mathrm{L})$ & -0.213 & 0.003 \\
$\mathrm{PA}(\mathrm{mg} / \mathrm{L})$ & -0.344 & 0.000 \\
TBiL $(\mu \mathrm{mol} / \mathrm{L})$ & 0.232 & 0.041 \\
$\mathrm{INR}$ & 0.128 & 0.000 \\
AST $(\mathrm{U} / \mathrm{L})$ & 0.357 & 0.000 \\
GGT $(\mathrm{U} / \mathrm{L})$ & 0.311 & 0.002 \\
ALP $(\mathrm{g} / \mathrm{L})$ & 0.212 & 0.004 \\
PT $(\mathrm{s})$ & 0.198 & 0.048 \\
SCr $(\mu \mathrm{mol} / \mathrm{L})$ & 0.358 & 0.435 \\
ALT $(\mathrm{U} / \mathrm{L})$ & 0.214 & 0.000 \\
APTT $(\mathrm{s})$ & 0.111 & 0.025 \\
TT $(\mathrm{s})$ & 0.125 & 0.032 \\
\hline
\end{tabular}

AlB, albumin; PA, prealbumin; TBil, total bilirubin; INR, international normalized ratio; AST, aspartate aminotransferase; GGT, gammaglutamyl transpeptidase; ALP, alkaline phosphatase; PT, prothrombin time; SCr, serum creatinine; ALT, alanine aminotransferase; APTT, activated partial thromboplastin time; TT, thrombin time.

${ }^{*} p<0.05$ or $* * p<0.01$ vs. normal value.

serological indicators from those 161 patients were substituted into the liver fibrosis scoring formula to calculate the $\mathrm{R}$ value. The $\mathrm{R}$ value was then compared to the Laennec liver fibrosis scoring system, and a grading standard for the liver fibrosis scoring formula was obtained.

Using matter-element analysis, the following liver fibrosis formula was created with the 8 preoperative serological indicators:

$$
\begin{aligned}
\mathrm{R}=\mathrm{a} \times \frac{\mathrm{ALB}-22.17}{49.56}+\mathrm{b} \times \frac{\mathrm{PA}-4.7}{202.6}+\mathrm{c} \times \frac{117.9-\mathrm{TBiL}}{113.98}+\mathrm{d} \times \frac{2.36-\mathrm{INR}}{1.56} \\
+\mathrm{e} \times \frac{304-\mathrm{AST}}{289}+\mathrm{f} \times \frac{774-\mathrm{GGT}}{759}+\mathrm{g} \times \frac{646-\mathrm{ALP}}{594} \\
+\mathrm{h} \times \frac{28.5-\mathrm{PT}}{17.7}
\end{aligned}
$$

In this formula, ALB, PA, TBiL, INR, AST, GGT, ALP, and PT represent the clinical levels of these 8 indicators. The weights are represented by a-h, and they were determined using matter-element analysis. From a-h, the determined values were $0.0739,0.1694,0.0519$, $0.0029,0.1467,0.1663,0.3527$, and 0.0362 . The preset data are from the 161 patients; 22.17 and 4.7 are the minimum detected values for ALB and PA, respectively, whereas $117.9,2.36,304,774,646$, and 28.5 represent the maximum clinical values for TBiL, INR, AST, GGT, ALP, and PT, respectively (Table 3 ). The values of the denominators in the formula were equal to the maximum values minus the minimum values. The value of $\mathrm{R}$ calculated using this formula will indicate the patient's stage of liver fibrosis.

\subsection{Degree of liver fibrosis according to the $R$ value}

Based on the $\mathrm{R}$ value according to the above formula, different degrees of liver fibrosis were identified: $i$ ) the value of $0.802 \leq \mathrm{R}<1$ indicates the early stage of liver cirrhosis, which corresponds to Laennec stages $0-3$; ii) the value of $0.752 \leq \mathrm{R}<0.802$ indicates the mild stage of liver cirrhosis, which corresponds to Laennec stage $4 \mathrm{~A}$; iii) the value of $0.698 \leq \mathrm{R}<0.752$ indicates the moderate stage of liver cirrhosis, which corresponds to Laennec stage $4 \mathrm{~B}$; and $i v$ ) the value of $0.444 \leq \mathrm{R}<$ 0.698 indicates the severe stage of liver cirrhosis, which corresponds to Laennec stage $4 \mathrm{C}$. In addition, $\mathrm{R}=1$ indicates normal liver tissue, representing well liver function.

\section{Discussion}

Liver cirrhosis is a disease that affects a massive number 


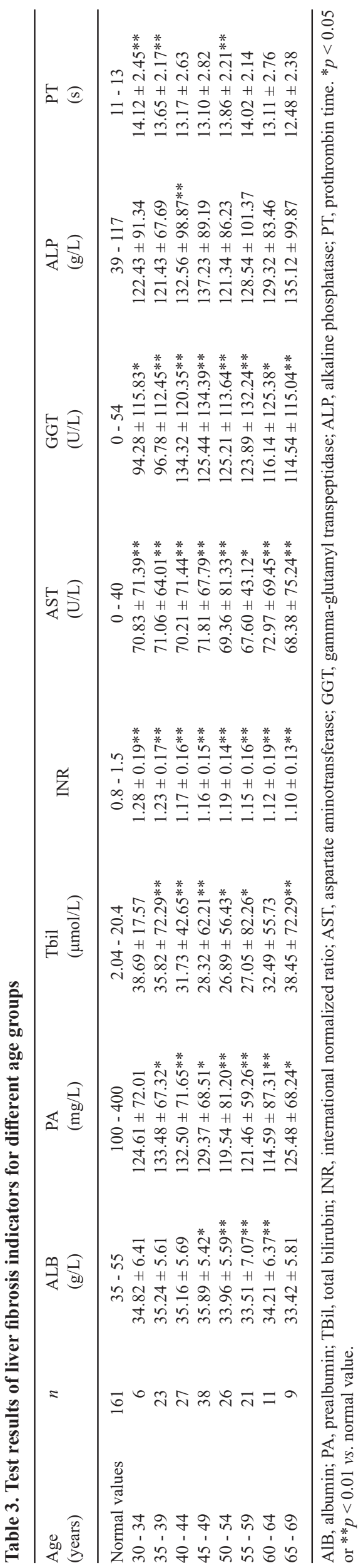

of patients worldwide. As this disease progresses, it is more difficult to cure clinically. Many cases of liver fibrosis develop into liver cirrhosis and even liver cancer due to delayed diagnosis. Therefore, liver cirrhosis needs to be properly diagnosed and staged.

A liver biopsy is still considered to be the "gold standard" for evaluating the severity of liver cirrhosis $(16,17)$. For example, the pathological grading of fibrosis in China is divided into four stages according to the prevention and treatment of viral hepatitis, and liver cirrhosis is the highest level. Nonetheless, longterm clinical practice has revealed that conditions differ in different patients with liver cirrhosis, challenging classification based on a liver biopsy. The traumatic consequences of liver biopsy are discouraging to most patients and could result in accidental deaths in extreme cases.

To overcome the aforementioned problems with liver biopsy, some studies have developed mathematical methods to evaluate liver function. The semi-quantitative Child-Pugh score is widely used to evaluate liver function, and it takes into account both clinical and biochemical parameters in patients with liver cirrhosis (18). Recent studies have also found that the MELD score was better at assessing the severity and prognosis of end-stage liver diseases than the Child-Pugh score (19-21).

Another important factor for evaluating the clinical treatment of liver cirrhosis is the stage. The Laennec liver fibrosis scoring system is widely used in clinical settings, and it divides liver cirrhosis into several levels according to the thickness of the fibrous septa and regenerative nodules. However, the Laennec system is not flawless. In some cases, stage $4 \mathrm{C}$ liver fibrosis was expected to improve to stage $4 \mathrm{~A}$ after anti-fibrosis therapy, but the grading system could not accurately identify that improvement, which could lead to false conclusions. Therefore, more accurate grading methods need to be devised to classify liver cirrhosis in more detail (22-25).

The liver has a variety of metabolic functions, and it also has a strong capacity for storage, compensation, and regeneration. When the liver is damaged to a certain extent, the amount of some substances synthesized and secreted by the liver or the activity of some enzymes in serum will be abnormal. Detection of these indicators can indicate liver damage and pathological changes. SFLI was obtained using matter-element analysis, and studies have indicated that its results agree with the Child-Pugh classification and also provide a more objective basis for early diagnosis, grading of liver function, and determination of the development of liver cirrhosis and its prognosis $(12,13)$. Liver fibrosis is a process of abnormal proliferation of connective tissue in the liver caused by various pathogenic factors. All liver injury and repair and healing processes cause fibrosis, which is accompanied by the expression or 
inhibition of fibrosis-related proteins. Therefore, the current study used matter-element analysis to create a formula based on serological indicators in order to facilitate preoperative evaluation of the degree of liver fibrosis in patients with HCC. This study also analyzed the relationship between the results of that formula and histological sub-classification of the Laennec system. The hope is to devise a simple and universally acceptable method that can indicate the degree of liver fibrosis in patients with $\mathrm{HCC}$ in a short of time following a clinical examination.

In conclusion, 8 preoperative serological indicators (ALB, PA, TBil, INR, AST, GGT, ALP, and PT) were retrospectively determined in 161 patients with HCC and different degrees of liver fibrosis to establish a formula using matter-element analysis. The hope is that such a formula for preoperative evaluation of the degree of liver fibrosis using non-invasive methods would be useful in the clinical diagnosis and treatment of patients with HCC in the future.

\section{Acknowledgements}

The authors wish to thank Dr. Xiao Tan and Professor Sen Liu (China Three Gorges University) for helpful discussions and thank the members of the Department of Pathology of Yichang Central Hospital for their outstanding technical assistance. This work was financially supported by China Three Gorges University (2015KZL14).

\section{References}

1. Schuppan D, Afdhal NH. Liver cirrhosis. Lancet. 2008; 371:838-851.

2. Schiff ER, Sorrell MF, Maddrey EC, editors. Schiff's Diseases of the Liver. 9th Edition Lippincott, Williams \& Wilkins; Philadelphia: 2003.

3. Sherlock S, Dooley J, editors. Diseases of the Liver and Biliary System. 11th Edition Blackwell Science; Oxford, UK; Malden, MA: 2002.

4. Bircher J, Benhamou JP, McIntyre N, Rizzetto M, Rodes J, editors. Oxford Textbook of Clinical Hepatology. 2nd Edition Oxford University Press; 1999.

5. Bravo AA, Sheth SG, Chopra S. Liver biopsy. N Engl J Med. 2001; 344:495-500.

6. Baranova A, Lal P, Birerdinc A, Younossi ZM. Noninvasive markers for hepatic fibrosis. BMC Gastroenterol. 2011; 11:91.

7. Wanless IR, Nakashima E, Sherman M. Regression of human cirrhosis. Morphologic features and the genesis of incomplete septal cirrhosis. Arch Pathol Lab Med. 2000; 124:1599-1607.

8. Wang W, Li J, Pan R, A S, Liao C. Association of the Laennec staging system with degree of cirrhosis, clinical stage and liver function. Hepatol Int. 2015; 9:621-626.

9. Kim SU, Oh HJ, Wanless IR, Lee S, Han KH, Park YN. The Laennec staging system for histological subclassification of cirrhosis is useful for stratification of prognosis in patients with liver cirrhosis. J Hepatol.
2012;57:556-563.

10. Almpanis Z, Demonakou M, Tiniakos D. Evaluation of liver fibrosis: "Something old, something new...". Ann Gastroenterol. 2016; 29:445-453.

11. Smith JO, Sterling RK. Systematic review: Non-invasive methods of fibrosis analysis in chronic hepatitis C. Aliment Pharmacol Ther. 2009; 30:557-576.

12. Zheng J, Xing RC, Zheng WH, Liu W, Yao RC, Li XS, Du JP, Li L. A comparative study on postoperative mortality prediction of SFLI scoring system and ChildPugh classification in patients with hepatocellular carcinoma. J BUON. 2017; 22:709-713.

13. Liu W, Zheng J, Xing R. Clinical significance of a scoring formula of liver injury for the preoperative evaluation of patients with liver cirrhosis. Eur J Gastroenterol Hepatol. 2014; 26:95-100.

14. Kim MY, Cho MY, Baik SK, Park HJ, Jeon HK, Im CK, Won CS, Kim JW, Kim HS, Kwon SO, Eom MS, Cha SH, Kim YJ, Chang SJ, Lee SS. Histological subclassification of cirrhosis using the Laennec fibrosis scoring system correlates with clinical stage and grade of portal hypertension. J Hepatol. 2011; 55:1004-1009.

15. Ding XJ, Li SB, Li SZ, Liu HS, Liu B, Xu FM, Gu RW. A quantitative of the relationship between levels of liver fibrosis markers in sera and fibrosis stages of liver tissues of patients with of chronic hepatic diseases. Zhonghua Gan Zang Bing Za Zhi. 2005; 13:911-914. (in Chinese)

16. Sebastiani G, Alberti A. Non-invasive fibrosis biomarkers reduce but not substitute the need for liver biopsy. World J Gastroenterol. 2006; 12:3682-3694.

17. Sebastiani G. Non-invasive assessment of liver fibrosis in chronic liver diseases: Implementation in clinical practice and decisional algorithms. World J Gastroenterol. 2009; 15:2190-2203.

18. Manley BJ, Tennenbaum DM, Vertosick EA, Hsieh JJ, Sjoberg DD, Assel M, Benfante NE, Strope SA, Kim E, Casuscelli J, Becerra MF, Coleman JA, Hakimi AA, Russo P. The difficulty in selecting patients for cytoreductive nephrectomy: An evaluation of previously described predictive models. Urol Oncol. 2017; 35(:35. e1-35.e5.

19. Nishikawa H, Osaki Y. Liver cirrhosis: Evaluation, nutritional status, and prognosis. Mediators Inflamm. 2015; 2015:872152.

20. Gallegos-Orozco JF, Vargas HE. Liver transplantation: From Child to MELD. Med Clin North Am. 2009; 93:931950.

21. Kamath PS, Wiesner RH, Malinchoc M, Kremers W, Therneau TM, Kosberg CL, D'Amico G, Dickson ER, Kim WR. A model to predict survival in patients with end-stage liver disease. Hepatology. 2001; 33:464-470.

22. Davis GL, Esteban-Mur R, Rustgi V, Hoefs J, Gordon SC, Trepo C, Shiffman ML, Zeuzem S, Craxi A, Ling MH, Albrecht J. Interferon alfa-2b alone or in combination with ribavirin for the treatment of relapse of chronic hepatitis $\mathrm{C}$. International Hepatitis Interventional Therapy Group. N Engl J Med. 1998; 339:1493-1499.

23. Lai CL, Chien RN, Leung NW, Chang TT, Guan R, Tai DI, Ng KY, Wu PC, Dent JC, Barber J, Stephenson SL, Gray DF. A one-year trial of lamivudine for chronic hepatitis B. Asia Hepatitis Lamivudine Study Group. N Engl J Med. 1998; 339:61-68.

24. McHutchison JG, Gordon SC, Schiff ER, Shiffman ML, Lee WM, Rustgi VK, Goodman ZD, Ling MH, Cort S, Albrecht JK. Interferon alfa- $2 \mathrm{~b}$ alone or in combination 
with ribavirin as initial treatment for chronic hepatitis $\mathrm{C}$ : Hepatitis Interventional Therapy Group. N Engl J Med. 1998; 339:1485-1492.

25. Shiratori $Y$, Imazeki F, Moriyama M, Yano M, Arakawa Y, Yokosuka O, Kuroki T, Nishiguchi S, Sata M, Yamada G, Fujiyama S, Yoshida H, Omata M. Histologic improvement of fibrosis in patients with hepatitis $\mathrm{C}$ who have sustained response to interferon therapy. Ann Intern Med. 2000; 132:517-524.

(Received November 30, 2018; Revised February 15, 2019; Accepted February 26, 2019) 